\title{
El interés por la sumillería en el siglo XIX: estudio del Manuel de sommelier ou instruction pratique sur la manière de soigner les vins de André Jullien
}

\author{
Francisco Luque Janodet \\ Universidad de Córdoba \\ 132lujaf@uco.es \\ https://dx.doi.org.10.12795/futhark.2017.il2.03
}

Fecha de recepción: 15.01.2017
Fecha de aceptación: 20.02 .2017

Resumen: El presente trabajo se ocupa de analizar la figura y obra de uno de los pioneros en el ámbito de la enología del siglo XIX: André Jullien, inventor de varios artilugios como la cannelle aérifère y autor de una de las obras más importantes sobre el estado de todos los viñedos del mundo, Topographie de tous les vignobles connus, así como de uno de los primeros manuales de sumillería, obra que centrará el presente artículo. En este proyecto, se analizará el surgimiento e interés que comienza a generar la sumillería a finales del siglo XIX. Se abordará, además, la figura de Jullien y su producción científica para ahondar en su aportación a la sumillería con el Manuel du sommelier, obra pionera en este campo.

Palabras clave: sumillería, enología, inventor.

\section{The interest in wine steward in the $19^{\text {th }}$ century: considerations about André Jullien's Manuel du sommelier ou instruction pratique sur la manière de soigner les vins}

\begin{abstract}
This work focuses in the figure and oeuvre of one of the pioneers in the field of enology of the 19th century: André Jullien, creator of some inventios like the cannelle aérifere and author of one of the most important treatises about agronomy: Topographie de tous les vignobles connus. He wrote also one of the first dissertations about wine tasting and sommelier. This paper will analyze the interest in sommelier in the end of $19^{\text {th }}$ century, the figure of André Jullien and its scientific inventios. It will be also analyzed the treatise Manuel du sommelier.
\end{abstract}

Key words: wine steward, enology, inventor. 
Sumario: I. Introducción. 2. Un acercamiento a la producción de documentos científico-técnicos en el ámbito de la enología y sumillería. 3. El Manuel du sommelier, ou instruction pratique sur la manière de soigne le vins de André Julliens. 4. Conclusiones.

\section{Introducción}

El vino es un producto que ha acompañado al ser humano a lo largo de la historia. Se trata de una bebida de gran influencia sociocultural en las distintas civilizaciones, como atestigua su presencia en la producción literaria y en las artes en general. En este sentido, no es de extrañar que el vino sea objeto de estudio desde distintas áreas del conocimiento, abarcando la sumillería, la enología, la historia del arte, la traducción y la terminología. La vitivinicultura es, además, uno de los ámbitos de la agronomía más estudiados desde hace siglos, existiendo numerosos tratados centrados en distintos aspectos como, por ejemplo, la mejora de las técnicas de poda y de los cuidados de la vid, así como la simplificación de los procesos de vinificación, de manera que permitan obtener un producto de mayor calidad en un período menor de tiempo y con una reducción sustancial del esfuerzo humano. Asimismo, especialmente a partir del siglo XIX, comenzamos a encontrar obras que abordan distintas temáticas relacionadas con el ámbito vitivinícola como la cata del vino, su terminología y la sumillería. De hecho, parte del interés del vino radica en el interés sociocultural de esta bebida, especialmente palpable en las culturas de la cuenca mediterránea, con ejemplos como el Libro de agricultura de Abú Zacarías Al-Awwan, escrito durante el período de dominación musulmana en la península lbérica en el siglo XIII, en el cual se consagran ciertas partes al cuidado de la vid y a los usos del vino. De esta manera, dentro de la historia de los estudios del vino, debemos distinguir, como señala Peynaud (1988), la «historia de la enología» de la «historia de la vinificación», dado que se encuentran en planos distintos y divergen en su antigüedad. De hecho, para este autor (ibid.), existe un «periodo pre-enológico» en el que la elaboración del vino estuvo al margen de la ciencia, y un periodo de «enología científica», contemporánea a «los procesos de la química y de las primeras teorías sobre las causas de la fermentación y las enfermedades de los vinos».

A partir de los siglos XVIII y XIX, con el surgimiento de la enología, se produce un aumento considerable de la bibliografía científica en torno a los cuidados de la vid y a la producción y conservación del vino, con ejemplos como L'art de faire le vin de Chaptal y, en español, el Arte de hacer y conservar el vino de Carbonell i Bravo. Asimismo, comenzamos a encontrar en el siglo XIX, una tendencia por estudiar los viñedos existentes en Francia y en el mundo, con casos como la Topographie de tous les vignobles connus (18I6) de André Jullien, en la cual el autor, tras haber realizado un estudio con datos estadísticos, presenta un 
compendio compuesto por una serie consideraciones previas, en las que incluye un vocabulario de términos técnicos del ámbito vitivinícola y de la cata del vino, una sección dedicada a los vinos de la antigüedad, así como un análisis del vino y de sus propiedades. La Topographie de Jullien cuenta con un total de 28 capítulos se dividen claramente en vinos de Francia y vinos extranjeros, como ya puso de manifiesto el propio autor (I8I6: XIX):

L'ouvrage étant divisé en deux parties, dont la première comprend les vignobles de France et la seconde ceux des pays étrangers, j'ai terminé chacune d'elles par une classification générale des vins dont il a été fait mention dans les divers chapitres. Cette portion de mon travail est sans doute la plus imparfaite et celle qui excitera le plus de réclamations de la part des propriétaires. Je les prie d'être persuadés que toutes les erreurs que j'ai pu commettre à ce sujet, sont involontaires, et que je m'empresserai de les rectifier dès qu'ils m'auront fait parvenir les renseignemens nécessaires pour assigner à leurs vignobles le rang qu'ils doivent occuper [...].

Por tanto, la primera parte está enteramente dedicada a los vinos franceses y compuesta por un total de 25 capítulos y un capítulo dedicado a la clasificación general de los vinos de Francia en vins rouges, vins blancs y vins de liqueur. La segunda parte está centrada en los vinos de los países extranjeros: el primer capítulo está dedicado a Suecia, Noruega y Dinamarca; el segundo al Reino Unido de Gran Bretaña e Irlanda; el tercero, al Reino de los Países Bajos y de Bélgica; el cuarto, a Alemania y Zollverein; el quinto, al Imperio Austríaco; el séptimo a Italia; el octavo, a España; le siguen los capítulos dedicados a Portugal, a Rusia y a Polonia, a Turquía, a Grecia y a las islas Jónicas. Asimismo, el capítulo XIII está dedicado a Asia, con subapartados que contemplan tanto regiones de Oriente Medio como del Lejano Oriente: Arabia, Persia, Afganistán, China o Japón; mientras que el capítulo XIV está dedicado a África $y$, el siguiente, a las islas del océano Atlántico y, finalmente, el capítulo XVI, a América. De la misma manera que en la primera parte de la obra, Jullien se propone hacer una clasificación general de los vinos extranjeros, a los que divide, de nuevo, en tres categorías: vins rouges, vins blancs y vins de liqueur. A este trabajo, debemos añadir los diez apéndices que elaboró Jullien mediante la consulta de documentos oficiales, como él mismo afirma (1866:530) y que completan la quinta edición de la obra, denominados: «Nombre d'hectares plantés en vigne en 1788, 1829 et 1847 », «Statistique de la production et de la consommation de vin», «Superficie des vignobles et production moyenne des vins blancs et rouges de 1852 à |869», «Production du vin de 1859 à |864», «Production de l'alcool en 1862, 1863, |864», «Production de la bière», «Prix des vins exportés en |826-|864», «Prix des vins importés aux mêmes années», «Alcoolisation des vins pour l'exportation» $y$, finalmente, «Dimensions que doivent avoir les futailles métriques». Cierra la obra la tabla general de vinos, constituida, de nuevo, por dos partes: una dedicada a los vinos de Francia y otra dedicada a los vinos foráneos. Jullien, en este 
sentido, se presenta como uno de los pioneros de este ámbito, aunque ya existía un precedente:

Le seul ouvrage qui en traite avec quelques détails a été récemment publié à Madrid, et vient d'être traduit en français par M. Caumels. Encore ne s'occupet-il que des vignes de l'Andalousie, dont il décrit cent-vingt variétés; l'auteur assure que l'Espagne en contient au moins cinq cents.

Hace referencia, concretamente a la obra de Simón Clemente Rojas Clemente y Rubio, que lleva por título Ensayo sobre las variedades de la vid común que vegetan en Andalucía, con un índice etimológico y tres listas de plantas en que se caracterizan varias especies nuevas, publicada por Gaspar Victoriano Suárez en Madrid en 1807. La versión en francés de Caumels, con el título Essai sur les variétés de la vigne qui végètent en Andalousie aparecería en 1814 en París editado por Poulet. Se trata, pues, de una línea de investigación en la que participarán otros autores como Cavoleau con su Oenologie française cuyo fin era conocer la extensión superficial de los vinos franceses, los productos, su precio, los lugares de exportación y su uso en el consumo interno o en el comercio exterior y el Étude des vignobles de France pour servir à l'enseignement mutuel de la viticulture et de la vinification françaises de Guyon (1868). Por ello, se puede afirmar que estas aportaciones se inscriben en una línea de investigación novedosa que comenzamos a encontrar en el siglo XIX, la cual permitió un aumento de tratados centrados en otras cuestiones como el Manuel du sommelier, ou instruction pratique sur la manière de soigner les vins $(18 \mid 3)$ de Jullien, que centrará el presente artículo.

\section{Un acercamiento a la producción de documentos científico-técnicos en el ámbito de la enología y sumillería}

La enología, como ciencia que estudia el vino en su conjunto, surge de un conjunto de saberes tradicionales y de innovaciones desarrollados a lo largo de los siglos y siempre desde una perspectiva interdisciplinar. Si nos remontamos a la antigüedad, podemos observar la estrecha relación entre el cristianismo y el vino, pero también entre los musulmanes de Al-Ándalus con esta bebida, hecho que se observa en el Libro de Agricultura del médico lb-al-Awan, datado en el siglo XIII, considerado como una de las obras más completas que versan sobre esta bebida y su cultivo, o Theatrum Sanitaris de Abdul Hassar-al-Muktar, datada del siglo Xl, en la que el autor defiende las virtudes medicinales de esta bebida (Segarra, 20I2: 31). Ahora bien, si avanzamos más en el tiempo, podemos encontrar diversos tratados como el Liber de vinis, que, como señala Rodríguez Guerrero (2013: 44), es un tratado del siglo XIV que contiene una serie de recetas de productos terapéuticos elaborados a partir del vino, entre siete y cincuenta, dependiendo de las copias. Su extensa tradición manuscrita e impresa, como señala este autor, permite valorarlo 
como un texto de éxito en la Baja Edad Media y el Renacimiento, con una difusión favorecida al médico valenciano Arnau de Vilanova, aunque, apunta, que «los especialistas actuales en el corpus médico de Arnau no lo reconocen como una obra auténtica» (ibid.) y es, actualmente, una obra de carácter anónimo. No obstante, como señala lbáñez Rodríguez (2015: 275) a partir de la segunda mitad del siglo XVIII comienzan a publicarse de forma más frecuente distintos escritos sobre el cultivo de la vid y la elaboración del vino, y, por tanto, la traducción de estas publicaciones no tardará tampoco en ser común. Este autor (ibid.) señala distintas publicaciones sobre el cultivo de la vid como el Traité de la culture des terres de Duhamel de Monceau (175I), coincidiendo, pues, con el surgimiento de las lenguas de especialidad. También recoge el Cours complet d'Agriculture ou dictionnaire universaire de Rozier (1800) y el L'Art de faire le vin (1807) de Chaptal. Posteriormente, en 1820 se publica el Arte de hacer y conservar el vino con una noticia acerca la fabricación del vinagre de Francisco Carbonell i Bravo, al que considera (20I5) como el primer manual de enología en español.

De hecho, tal es la importancia del estudio de la vid y el vino que lbáñez (2015: 275) compiló un corpus de 128 publicaciones centradas en esta temática, donde también incluye obras de agricultura, siempre y cuando recojan información sobre el cultivo de la vid y la elaboración del vino, en las que comprende tanto libros, tratados, artículos y opúsculos. El estudio de Ibáñez (20I5) demuestra que un total de 42 documentos $(32,81 \%)$ son traducciones y 86 son originales $(67,19 \%)$. Se trata de unos resultados novedosos, ya que la enología se constituye como un ámbito poco estudiado a lo largo del tiempo, si nos remitimos a las investigaciones de García Hurtado (1999b) este autor señaló que, en el siglo XVIII, el $31,7 \%$ de los libros traducidos pertenecían al ámbito religioso, un 19,2\% de las obras eran de temática literaria y un 10\% pertenecían al ámbito científico-técnico, mayoritariamente al campo de la medicina.

Ahora bien, a pesar de que el servicio de vinos es una temática que ha generado una cierta producción científica, hallándose la palabra sommelier, ya relacionada con el vino, registrada en el Dictionnaire royal de François Pomey, donde se define sommelier (162I) como una persona «qui a soins de la dépense du vin dans une maison» y la sommelerie como «charge du sommelier» y «le lieu où le sommelier distribuë le vin». Asimismo, el Dictionnaire François contenant généralement tous les monts tant vieux que nouveaux de Pierre Richelet (I706: 769), define esta oficio como: «celui qui a soin de la dépense du vin», mientras que la sommelerie es «le lieu dans une maison de qualité où l'on garde le vin de table».

Si bien es cierto que el ámbito del servicio de vinos comienza con obras como Le maistre d'hostel qui apprend l'ordre de bien seruir sur table \& d'y ranger les services ; ensemble Le sommelier qui enseigne la maniere de bien plier le linge en plusieurs figures et à faire toutes sortes de confitures, tant seiches que liquides 
comme aussi toutes sortes de dragées \& autres gentillesses fort utiles à toute le monde de Pierre David ( 1659), no será hasta el siglo XIX y principios del siglo XIX cuando comencemos a ver manuales y tratados centrados en la sumillería y el servicio de vinos como el manual ya citado de Jullien, el Nouveau manuel du sommelier: contenant l'art de soigner et de conserver les vins -donde también se incluye un vocabulario de términos especializados del ámbito de la sumillería titulado "Vocabulaire raisonné à l'usage des sommeliers » de Mardelin (1836) ; el Nouveau Manuel complet du sommelier et du marchand de vins contenant des notions succinctes sur les vins rouges, blancs et mousseaux de Maigne (192I) y la revista especializada Le sommelier. Revue mensuelle oficielle de l'Union des Sommeliers editada de 1923 a 1934.

De hecho, en el siglo XIX comenzamos a observar los primeros intentos de estandarización y homogeneización del vocabulario de la cata del vino. Jullien, quien presenta, en la primera edición de su Topographie de tous les vignobles connus (I8I6), aproximadamente cincuenta términos de este ámbito en el «Vocabulaire des termes techniques les plus fréquemment employés pour désigner les qualités, les défectuosités et les altérations des vins», que sería ampliado a lo largo de las distintas ediciones hasta llegar a las noventa entradas en la edición póstuma de 1866, siendo este autor como un pionero en el ámbito de la enología y de la sumillería. Como ya hemos señalado, a partir de este momento comienzan a presentarse proyectos de este tipo, como el ya citado « Vocabulaire raisonné à l'usage des sommeliers » de Mardelin (1836) donde se ofrecen sesenta términos que abarcan desde el ámbito de la cata (goût de pousse, vin muet), como de otros como del proceso de producción (fleur, soutirage des vins) e incluso del ámbito de la tonelería (gravele, jable, jauge, jauger, soufrage des tonneaux et des vins).

\section{El Manuel du sommelier, ou instruction pratique sur la manière de soigner les vins de André Jullien}

Existen pocos escritos fiables sobre la biografía de este autor, aunque sí aparece mencionado en la obra Biographie universelle, ou Dictionnaire de tous les hommes qui se sont fait remarquer par leurs écrits, leurs actions, leurs talents, leurs vertus ou leurs crimes, depuis le commencement du monde jusqu'à ce jour; d'après la Biographie universelle historique de Weiss ... etc., etc. par une société de gens de lettres (I847) de Feller donde se indica que Jullien nació en 1766 en Châlons-sur-Saône y se dedicó al negocio de vinos. Asimismo, parece ser que adquirió gran prestigio por sus descubrimientos y por sus aportaciones a la carrera industrial con obras como Appareils perfectionnés, propres transvaser les vins et autres liqueurs avec ou sans communication avec l'aire extérieur avec une plance représentant les appareils (1809). Sus primeros ensayos obtuvieron el favor de Chaptal, ministro del interior 
de Francia, llegando a dedicarle la presente obra y, asimismo, desarrolló distintos inventos como como les cannelles aérifères, cilindros mediante los que se trasvasa el vino a las botellas para clarificar este producto. André Jullien falleció en 1832 de cólera en París tras haber obtenido por sus inventos diversas medallas en exposiciones de productos industriales, así como la medalla de oro de la Académie de Sciences, el conocido como Grand Prix Montyon, con la que se galardonó su Topographie de tous les vignobles connus.

La obra que centrará el presente artículo se presenta en la quinta edición de I836 con el título Manuel du sommelier, ou instruction pratique sur la manière de soigner les vins contenant la théorie de la Dégustation, de la Clarification, du Collage et de la Fermentation secondaire des vins; les moyens de prévenir leur altération et de les rétablir lorsqu'ils sont dégénérés ou naturellement défectueux, de distinguer les vins purs des vins mélangés, frelatés ou artificiels, etc. suivi du tarif des droits de mouvement, d'entrée, d'octroie et de vente en détail, etc. En la portada de dicha edición se ofrece una breve reseña biográfica del autor: «Par. M. A. Jullien, Marchand de vin en gros, auteur de la Topographie de tous les vignobles connus, inventeur des poudres pour clarifier les vins, des cannelles aérifères et de plusieurs autres instrumens», con una dedicatoria a «M. Le Compte Chaptal, Pair de France, Membre de l'Académie des sciences, auteur de l'ouvrage intitulé : l'Art de faire le vin». En este caso, no debemos olvidar que Chaptal, conde de Chanteloup y como antiguo ministro del interior, apoyó los primeros escritos de este autor, de ahí la presente dedicatoria que ya apareció en la primera edición de 1813 .

La lectura del prefacio de la obra ofrece al lector una cantidad importante de información. En este caso, encontramos la exposición de motivos que llevó al autor a redactar esta obra en concreto:

Les vins les mieux soignés sont sujets à des altérations qui leur sont naturelles; quelquefois ils se rétablissent d'eux-mêmes, et presque toujours lorsqu'on y porte le remède nécessaire. Les personnes qui ne connaissent pas ces phénomènes croit que leur vin est perdu aussitôt qu'il prend un goût désagréable ; elles s'empressent de le vendre à vil prix, tandis que quelques mois plus tard elles auraient pu le boire pourvu de toutes ses qualités.

Témoin des pertes que le consommateur éprouve par la négligence qu'on apporte dans la direction de sa cave, j'ai pensé qu'il était utile de l'éclairer et de lui donner des moyens et des règles sûrs pour la conservation de ses vins, en lui indiquant en même tems les causes qui contribuent à les altérer. Tel est le but de ce petit ouvrage qu'on peut considérer comme un traité pratique à la portée de toutes les classes de la société. (Jullien, 1813 : vi-vii.)

No obstante, en lo que respecta a su finalidad última, Jullien (ibid.) argumenta que: 
Je n'ai point eu la prétention de remonter aux causes physiques qui influent sur la qualité des vins; ce sujet a déjà été traité par des savans distingués ; je me suis sur-tout attaché à être clair et concis, et à ne rien omettre de ce qui peut contribuer à la conservation et à l'amélioration d'une liqueur dont ont fait presque généralement usage en France.

Jullien presenta brevemente la estructura de la obra e incide en la existencia de un apartado dedicado a uno de sus instrumentos, los entonnoirs aériferes:

J'indique les méthodes les plus simples et les plus fréquemment employées pour conserver les vins, les éclaircir, les mettre en bouteilles, et remédier aux diverses altérations, soit naturelles, soit accidentelles, auxquelles ils sont exposés. Je passe ensuite à la description et à l'usage de mes cannelles aérifères pour transvaser les vins en bouteilles, ainsi que de mes entonnoirs aérifères servant à remplir toute espèce de vase à orifice étroit sans répandre de liquide et à filtrer les liqueurs sans évaporation. De nombreuses expériences ont constaté l'utilité de cette invention, qui a été honorée du suffrage de la Société d'Encouragement. Je termine cet opuscule par la description de mes cannelles doubles et de mes syphons aérifères, propres à la décantation des fluides éthérés et fétides sans répandre d'odeur dans l'atmosphère. Quoique ce chapitre soit étranger au sujet que je traite, je suis persuadé que ces instrumens peuvent recevoir des applications utiles; c'est pourquoi j'ai cru devoir leur accorder une place dans cet ouvrage. Je m'estimerai très-heureux que mon travail puisse être jugé digne de l'approbation du public ; je n'ai rien négligé pour le rendre aussi complet qu'il m'a été possible, et j'y ai ajouté tous les éclaircissemens que mon commerce m'a mis à portée de recueillir. (Jullien, 1813 : vii-viii).

Asimismo, en el prólogo de la quinta edición de 1836, Jullien señala las diferentes fuentes que le han permitido redactar el manual:

Les jeunes gens qui entreprennent le commerce des vins y puiseront des connaissances qu'ils ne pourraient acquérir que par plusieurs années d'expérience, et les négocians les plus expérimentés y trouveront encore quelques renseignemens et des recettes utiles qu'ils ne connaissaient pas. - Les ouvrages que j'ai consultés, et les relations que j'ai entretenues avec des propriétaires et des négocians de différens pays, pour obtenir les documens nécessaires à la rédaction de la Toppgraphie de tous les vignobles connus, qui est à sa troisième édition, m'ont fourni des instructions intéressantes pour le MANUEL DU SOMMELIER ; I'on trouvera donc cette cinquième édition beaucoup plus complète que les quatre premières. (Jullien, $1836: 6-7$ ).

Jullien se caracteriza, además, por mejorar y actualizar constantemente sus obras, algo que se puede observar a continuación:

Tous les chapitres ont subi des corrections et des augmentations plus ou moins considérables. J'ai ajouté plusieurs procédés peu connus, pour prévenir l'altération des vins et pour les rétablir lorsqu'ils sont altérés ou naturellement 
défectueux. Celui qui traite de l'expédition, indique toutes les précautions que l'on doit prendre pour éviter les avaries, les différentes manières de conditionner les tonneaux, les caisses et les paniers pour les garantir de l'infidélité des voituriers, ainsi qu'un moyen récemment employé avec succès pour préserver de la chaleur les vins en bouteilles qui doivent traverser la zone torride. La théorie et la pratique de la clarification ont reçu de nouveaux développemens dans le chapitre qui leur est consacré. [...] Le chapitre relatif aux vins sophistiqués a été augmenté, et j'en ai consacré un particulier à la litharge, poison très subtil dont on accuse le commerce de faire usage. Les tarifs des droits de circulation, d'entrée, d'octroi et de vente des boissons ont été rectifiés d'après les dernières lois rendues sur cette matière. J'y ai ajouté le tableau de comparaison de l'aréomètre centigrade à celui de Cartier, pour l'appli cation de l'échelle centésimale des degrés d'alcool aux eaux-de-vie et aux esprits; du thermomètre centigrade à celui de Réaumur; enfin des tables de conversion des litres en pintes ou en veltes, ou de celles-ci en litres. Les instrumens ordinairement employés à la manutention des vins n'étant pas les mêmes dans tous pays, j'ai pensé que les personnes qui mettent quelque importance à la bonne tenue de leurs caves, seraient satisfaites de connaître ceux qui présentent le plus de facilité dans leur emploi, et dont l'usage garantit le mieux des pertes et des avaries auxquelles cette liqueur est exposée. J'en ai, en conséquence, fait graver la collection sur une planche particulière, et j'en ai donné la description avec la manière de s'en servir. (Jullien, $1836: 7-10$ ).

La macroestructura de la obra de Jullien se encuentra dividida, además, en 20 capítulos, cada uno de los cuales está centrado en un aspecto particular: la recepción del vino, las tasas de movimiento de las bebidas, la disposición que debe tener una buena bodega y cómo solventar algunos inconvenientes de las bodegas; cómo preparar un barril para que ser llenado por primera vez de vino, cómo conservar los toneles vacíos que ya han servido, cómo verter el vino en los barriles, cómo fermentar el vino; el buqué de los vinos, la mezcla de vinos, las alteraciones de este producto y, en los capítulos 18, 19 y 20, presenta varias de sus creaciones que cree que serán útiles al bodeguero: la cannelle aérifère, el portebouteille, el entonnoir aérifere, el grand entonnoir aérifère y el entonnoir aérifêre double.

La quinta edición cuenta con un total de 29 capítulos, con algunos adicionales como «De la litarge employée pour préparer ou pour adoucir les vins» o «Droits établis sur les boissons par la loi du28 avril 1816, avec les modifications y apportées $\S$ ler Droits de circulation des boissons dans l'intérieur du royaume». De hecho, este aumento de capítulos se traduce en un aumento considerable de la extensión de la obra: de 180 en la edición de 1813 a 305 en aquella de 1836 . Asimismo, Jullien presenta a lo largo de las ediciones una «Table raisonnée des matières» que, en la primera edición contaba con 90 entradas, frente a las 193 de la quinta edición. 
Uno de los apartados más interesantes se encuentra en el capítulo XXIII en el que Jullien presenta la cannelle aérifere y otros instrumentos para trasvasar el vino y los licores en botellas o en damajuanas y que, según el propio autor (1836: 192), le valieron medallas $\mathrm{y}$ otros premios:

Admis à l'exposition des produits de l'industrie en 1819, avec mes poudres pour clarifier les vins, il m'a été accordé une médaille pour ces divers objets. En 1825, il m'a été accordé une nouvelle médaille pour mes poudres et un rappel de médaille pour mes instrumens, et ces récompenses m'ont été confirmées aux expositions de 1827 et 1854 .

Jullien, preocupado por el riesgo de falsificaciones que pudieran existir, afirma haber registrado la patente de su creación:

Je me suis assuré la propriété de cette découverte en prenant un brevet d'invention. La construction de ces instrumens exigeant des soins qu'on obtient rarement des ouvriers, je visite et essaie moi-même tous ceux que je livre au con sommateur. Ils sont garnis d'un écusson portant en relief : Par brevet d'invention, A. Jullien, Par ce moyen, ils ne seront pas confomdus avec les contrefaçons qui pourraient en être faites. (Jullien, 1836: 192)

La cannelle aérifère es un invento que el propio Jullien define como un instrumento:

[...] composée de deux pièces, dont l'une est une cannelle $i$ n o destinée à l'écoulement du liquide, et l'autre un tube recourbé $h$, garni d'un robinet $m$, par lequel l'air qui doit remplacer la liqueur, s'introduit dans la bouteille. Ces deux pièces, soudées ensemble et réunies par une grille en fer-blanc o, traversent un bouchon conique $g(\mathrm{I})$, propre à boucher l'orifice des bouteilles de tous calibres. II est évident que, si on place cette cannelle dans le col d'une bouteille A (fig. 2), tandis que le vin coulera par la cannelle, l'air s'introduira librement par le tube aérifère, et remplira le vide qui se fera dans la bouteille sous le point $d$ : alors le dépôt ne sera pas dérangé et ne pourra pas se mêler avec la liqueur. Les cannelles aérifères ordinaires servent pour toutes les bouteilles françaises, soit de la forme de Sèvres, soit de celle de Bordeaux ou de Champagne, ou même pour les bouteilles anglaises; mais j'ai été obligé de faire des cannelles d'une dimension particulière pour les bouteilles hollandaises, dont le col est très alongé et fort étroit, et pour les damesjeannes. Les cannelles aérifères, pour les bouteilles de toutes formes, coûtent $9 \mathrm{fr}$. en cuivre; $10 \mathrm{fr}$. 50 c. en cuivre étamé; et $75 \mathrm{fr}$. en argent. Celles pour les dames-jeannes coûtent 10 et 12 fr., suivant leurs dimensions.

Cabe señalar, además, la buena acogida que tuvo su obra en Europa, con traducciones a inglés, con el título The Wine Merchant companion and Butler's manual containing the best information of the selection and management of French wines (1825), con un prefacio de W. H. Hilton, a quien presuponemos traductor de la obra en inglés; en alemán, Der erfahrne Weinkellermeister: enthaltend 
gründliche Belehrungen über die Bereitung des Weines, nebst Anweisung, die Weine zu bearbeiten und im Keller zu behandeln, sie zu prüfen, zu versenden, sowohl in Fässern als in Kisten und Körben, sie aufzufüllen und abzuziehen, zu würzen, zu mischen, zu schönen ; von den Krankheiten und Ausartungen der Weine ; von den Vorrichtungen zur Behandlung schäumender Weine ; Wein- und Flüssigkeitsmaße der verschiedenen Länder und Städte : Beschreibung und Abbildung aller zum Weingeschäft nöthigen Geräthschaften, u. dgl. m. ; für einen Jeden, der Wein bereitet, bearbeitet oder Handel damit treibt (1836), traducido por Eduard Knossnagrl ; y a italiano con el título Manuale del cantiniere ovvero istruzioni pratiche sulla maniera di governare $\mathrm{i}$ vini contenente La Teoria dell'Assaggiatura, della Chiarificazione, della Concia alla colla, traducida por Pietro Nosetti y publicada en 1830 .

\section{Conclusiones}

Dedicado a su profesión, a la invención de artefactos relacionados con el vino y a la búsqueda de datos para actualizar las obras, André Jullien se puede considerar como un pionero en el ámbito de la cata y de la sumillería, no solo por haber creado el primer glosario monolingüe dedicado a la cata del vino, sino también por haber escrito uno de los primeros manuales para sumilleres, en el que trataba tanto la producción del vino, como su servicio y cata y en el que presenta varios de sus inventos, como la cannelle aérifere, de la que obtuvo una patente y cuya aplicación desarrolló en esta obra. Jullien realiza un esmerado y concienzudo trabajo a lo largo de los años, cuya importancia se puede observar en la recepción de sus obras en el continente europeo, aunque, desgraciadamente no llegaron nunca a España, a pesar de la dependencia de este país a los avances y progresos obtenidos en otras naciones, de entre las que destaca Francia, pues no debemos olvidar que este país fue cuna de la enología y el francés su ligua franca.

\section{Referencias bibliográficas}

Álvarez, M. (2015). Análisis de la terminología del vino en los Fabliaux. En: Balbuena, M. C. y Álvarez, M. (eds.): Lenguajes especializados y traducción: vitivinicultura, 5-18. Córdoba: Ediciones Don Folio.

Carbonell, F. (I820). Arte de hacer y conservar el vino con una noticia acerca la fabricación del vinagre. Barcelona: Antonio Brusi impresor.

Cavoleau, M. (1827). Oenologie française ou statistique de tous les vignobles et de toutes les boissons vineuses et spiritueuses de la France. París: Madame Huzard (née Vallat La Chapelle) Libraire.

Chaptal, J. A. C. (1807). L'art de faire le vin. París: Deterville. 
Cubero, J. I. (2003). El libro de agricultura de Al Awan. Volumen I y II. Sevilla: Junta de Andalucía. Viceconsejería. Servicio de Publicaciones y Divulgación.

David, P. (1659). Le maistre d'hostel qui apprend l'ordre de bien seruir sur table \& d'y ranger les services; ensemble Le sommelier qui enseigne la maniere de bien plier le linge en plusieurs figures et à faire toutes sortes de confitures, tant seiches que liquides comme aussi toutes sortes de dragées \& autres gentillesses fort utiles à toute le monde. París: Pierre David.

De Feller, F. X. (1848). Dictionnaire historique: ou, Biographie universelle de tous les hommes qui se sont fait remarquer par leurs écrits, leurs actions, leurs talents, leurs vertus ou leurs crimes, depuis le commencement du monde jusqu'à ce jour; d'après la Biographie universelle historique de Weiss ... etc., etc. par une société de gens de lettres. París, J. Leroux.

De Monceau, D. (1758). Traité de la culture des terres avec figures en taille-douce contenant les Expériences \& Réflexions sur la culture des terres \& sur la conservation des Grains, faires pendant les années 1755 \& I756. París: Hippolyte-Louis Guerin \& Louis-François Delatour.

Eurrutia, M. (2010). Sémantisme autour du vin : représentations symboliques et lexiculturelles. Anales de Filología Francesa I8: 183-202.

García, M. R. (1999b). Traduciendo la guerra. Influencias extranjeras y recepción de las obras militares francesas en la España del siglo XVIII. A Coruña, Universidade da Coruña, Servicio de Publicacións.

Ibáñez, M. (2015). La traducción científico-técnica francés-español en el ámbito de la enología (I750-1850). Çedille: Revista de Estudios Franceses II : 273-3 I I.

Guyon, J. (I868). Étude des vignobles de France pour servir à l'enseignement mutuel de la viticulture et de la vinification françaises. París: Imprimerie Imériale.

Jullien, A. (1809). Appareils perfectionnés, propres transvaser les vins et autres liqueurs avec ou sans communication avec l'aire extérieur avec une plance représentant les appareils. París: Arthus-Bertrand.

(1813/1836). Manuel du sommelier, ou Instruction pratique sur la manière de soigner les vins. París, D. Colas, Imprimeur-Libraire.

- (1816/1866). Topographie de tous les vignobles connus, contenant: leur position géographique, l'indication du genre et de la qualité des produits de chaque cru, les lieux où se font les chargements et le principal commerce de vin, le nom et la capacité des tonneaux, les mesures en usage, les moyens de transport ordinairement employés, suivie d'une classification générale des vins. París, Madame Huzard, imprimeur-libraire.

(1825). The Wine Merchant companion and Butler's manual containing the best information of the selection and management of French wines. Londres: G. Schulze.

- (I830) Manuale del cantiniere ovvero istruzioni pratiche sulla maniera di governare $i$ vini contenente La Teoria dell'Assaggiatura, della Chiarificazione, 
della Concia alla colla [Traducción de Pietro Nosetti]. Turín: Fratelli Reycend e Comp.

(1836). Der erfahrne Weinkellermeister: enthaltend gründliche Belehrungen über die Bereitung des Weines, nebst Anweisung, die Weine zu bearbeiten und im Keller zu behandeln, sie zu prüfen, zu versenden, sowohl in Fässern als in Kisten und Körben, sie aufzufüllen und abzuziehen, zu würzen, zu mischen, zu schönen ; von den Krankheiten und Ausartungen der Weine ; von den Vorrichtungen zur Behandlung schäumender Weine ; Wein- und Flüssigkeitsmaße der verschiedenen Länder und Städte : Beschreibung und Abbildung aller zum Weingeschäft nöthigen Geräthschaften, u. dgl. m. ; für einen Jeden, der Wein bereitet, bearbeitet oder Handel damit [Traducción de Eduard Knossnagel]. Leipzig: Gottfr. Baffe.

Lamelin, E. (I630). L'avant goust du vin. Déclaration de san ature, faculté medicinale et alimentaire. La manière de préparer les vins artificiels. Douay: lean de Fampoux.

López Alejandre, M. M. (2000). Los vinos de Montilla-Moriles. Junta de Andalucía. Consejería de Agricultura y Pesca. Sevilla: A. G. Novograf

Maigne, P. (192I): Nouveau Manuel complet du sommelier et du marchand de vin contenant des notions succinctes sur les vins rouges, blancs et mousseux, leur classification par Vignobles et par Crus, l'art de les déguster, la description du Materiel de Cave, les Soins à donner aux Vins en cercles et en bouteilles, le Traitement des Vins malades ou altérés, les Coupages, les moyens de reconnaître les Falsifications. París: L. Mulo.

Mardelin, M. (1836). Nouveau manuel du sommelier: contenant l'art de soigner et de conserver les vin, de les préserver et de les guérir des diverses maladies auxquelles ils sont exposés; la Nomenclature de tous les Vins français et étragers ; un Vocabulaire raisonné à l'usage du sommelier ; le tout précédé d'une notice sur l'art de faire les vins. París: Au dépot des nouveaux manuels.

Peynaud, E. (1988). Le vin et les jours. París: Bordas.

Pomey, F. (162I). Le petit Dictionnaire royal françois-latin. Lyon: Antoine Molin.

Richelet, P. (1706). Dictionnaire francois: contenant generalement tous les mots tant vieux que nouveaux, et plusieurs remarques sur la langue francoise; ses expressions propres, figure'es et burlesques, la Prononciation des Mots les plus dificiles, le Genre des Noms, la Conjugaison des Verbes, leur régime, celui des Adjectifs \& des Prépositions, Avec les Termes les plus connus des Arts \& des Sciences. Ámsterdam: Jean Elzevir.

Rodríguez, J. (2013). El Origen del Pseudo-arnaldiano Liber de vinis, Obra del magister Silvester (ca. 1322-1328), y su Tradición Manuscrita en el Siglo XIV. Azogue 7: 44-74. 
Rojas, S. C. (1807): Ensayo sobre las variedades de la vid común que vegetan en Andalucía, con un índice etimológico y tres listas de plantas en que se caracterizan varias especies nuevas. Madrid, Imprenta de Villalpando.

(I8|4). Essai sur les variétés de la vigne qui végètent en Andalousie. [Traducción del marqués de Caumels]. París: Poulet.

Rozier, F. (1838). Nouveau cours complet d'Agriculture du XIXe siècle, contenant la théorie et la pratique de la grande et de la petite culture, l'économie rurale et domestique, la médecine veterinaire, etc., ou Dicionnaire raisonné et universel d’agriculture. París : Libraireie encyclopédie de Roret.

Segarra, O. (20I2). La cultura del vino. Barcelona: Amat.

Union des sommeliers de Paris : Le sommelier. Revue mensuelle oficielle de l'Union des Sommeliers. París. 